\title{
Modélisation numérique du comportement non-linéaire d'un pieu chargé latéralement avec prise en compte du cisaillement à l'interface des couches de sable.
}

\section{S. Belkhir}

Doctorant, Laboratoire de Mécanique, G.R.G.C, Université du Havre

Quai Frissard B.P.265 - 76055 Le Havre Cedex, France.

D. Levacher

Professeur des Universités, Laboratoire de Mécanique, G.R.G.C, Université du Havre, Quai Frissard B.P.265 - 76055 Le Havre Cedex, France.

W. Hamadeh

Enseignent - chercheur

S. Mezazigh

ATER à l'IUT du Havre, Laboratoire de Mécanique, G.R.G.C, Université du Havre, Quai Frissard B.P.265 - 76055 Le Havre Cedex, France.

\section{Résumé}

Du fait des progrès réalisés dans la compréhension des mécanismes qui régissent le comportement des fondations profondes, on admet maintenant que les pieux verticaux puissent résister à des efforts latéraux.

Cet article présente un modèle de calcul numérique du comportement non-linéaire d'un pieu chargé latéralement avec prise en compte ou non du cisaillement à l'interface des couches du sable, en s'appuyant sur les méthodes basées sur l'utilisation des courbes p-y. Plusieurs exemples sont traités à l'aide du modèle et comparés aux résultats des essais obtenus sur des modèles de pieux réduits centrifugés. Une comparaison entre deux codes de calcul numérique est aussi proposée.

\section{Introduction}

Les fondations profondes sur pieux, très largement utilisées dans la construction des ouvrages, connaissent un développement croissant. Les progrès réalisés dans les méthodes de dimensionnement, les innovations technologiques dans la réalisation des pieux, la qualité de plus en plus médiocre des terrains laissés aux constructeurs et les dimensions importantes des ouvrages sont à l'origine de ce développement. Les approches théoriques sont, à l'heure actuelle, insuffisantes pour tenir compte de tous les paramètres d'interaction sol-pieu. Par exemple, les méthodes existantes ne prennent pas toutes en compte le cisaillement à l'interface des couches du sol. Par ailleurs, l'obtention de données expérimentales sur site pour le problème du chargement latéral est beaucoup plus délicate et onéreuse que pour celui du chargement axial. La nécessité, aujourd'hui encore d'étudier l'influence de divers paramètres, a amené bon nombre de chercheurs à utiliser des 
modèles réduits. Dans la bibliographie, on trouve peu de modèles de calcul nonlinéaires sur ce sujet, et seulement pour des configurations simples.

Cet article est consacré à la présentation succincte d'un modèle numérique, basé sur la méthode de la matrice transfert, applicable à des pieux chargés latéralement avec prise en compte ou non du cisaillement à l'interface des couches du sable. On illustre ensuite les possibilités du modèle sur quelques exemples de pieux chargés latéralement dont les résultats sont ensuite confrontés avec ceux du programme PILATE - LCPC (Frank et col.,1990), et avec ceux des essais sur des modèles de pieux réduits centrifugés.

\section{Modélisation numérique}

\section{Modèle de Pasternak (1954)}

Le comportement du sol étant extrêmement complexe, son étude a conduit à l'utilisation de divers modèles.

Pasternak (1954) a proposé un modèle de comportement du sol en tenant compte de l'existence d'une interaction de cisaillement entre les ressorts. Ceci est obtenu en reliant les ressorts à une couche d'éléments incompressibles qui peuvent subir uniquement des déformations tangenitielles dues au cisaillemeñt (Figure 1).

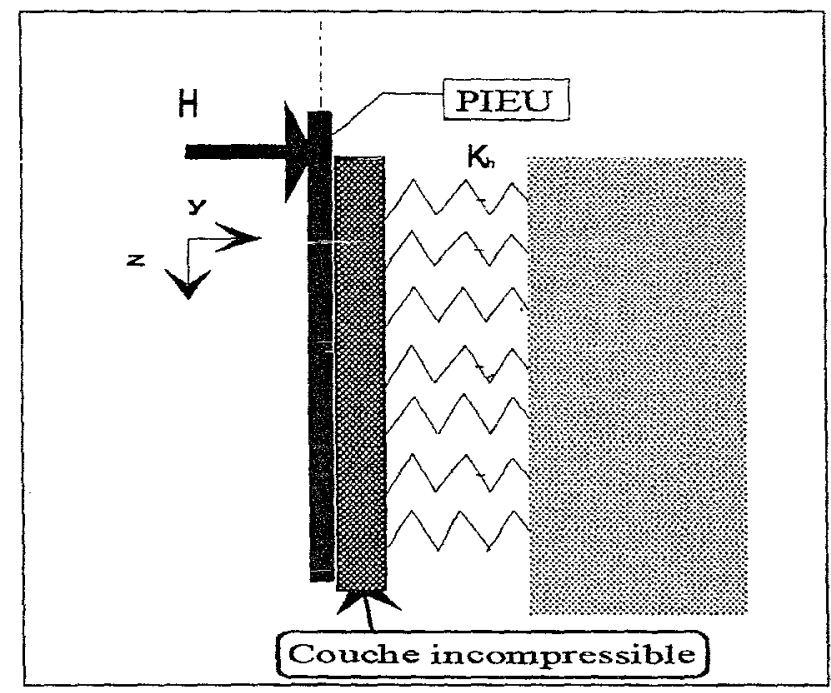

Figure 1 : Modèle de Pasternak

La couche de cisaillement est supposée isotrope avec un module de cisaillement $\mathrm{G}_{\mathrm{s}}$. La loi de réaction est écrite sous la forme suivante:

$$
p=K_{h} y+G_{s} \nabla^{2} y
$$

avec 


$$
\nabla^{2}=\frac{\partial}{\partial \mathrm{x}^{2}}+\frac{\partial}{\partial \mathrm{z}^{2}}
$$

Dans le cas d'un pieu chargé latéralement, le déplacement latéral n'est fonction que de la profondeur $z$, et l'équation (1) peut s'écrire sous la forme suivante :

$$
\mathrm{p}=\mathrm{K}_{\mathrm{h}} \mathrm{y}+\mathrm{G}_{\mathrm{s}} \frac{\mathrm{d}^{2} \mathrm{y}}{\mathrm{d} \mathrm{z}^{2}}
$$

Ce modèle est intéressant, puisqu'il tient compte d'une interaction de cisaillement entre les ressorts et propose une loi de réaction du sol fonction des modules de réaction et de cisaillement. Nous avons donc utilisé ce modèle dans notre étude du comportement du pieu chargé latéralement.

\section{Détermination du module de cisaillement du sable}

Seed et al. (1986) ont exprimé le module de cisaillement maximal du sable en fonction de l'indice de densité $\left(I_{D}\right)$ et du poids volumique effectif $(\gamma)$, sous la forme :

$$
\mathrm{G}_{\max }=21,7\left(\mathrm{~K}_{2}\right)_{\max } \mathrm{P}_{\mathrm{a}}\left(\frac{\sigma_{\mathrm{m}}^{\prime}}{\mathrm{P}_{\mathrm{a}}}\right)^{0,5} \quad\left(\mathrm{KN} / \mathrm{m}^{2}\right)
$$

avec

$\sigma_{m}^{\prime}=\gamma^{\prime} \cdot z$

$\mathrm{P}_{\mathrm{a}}$ : pression atmosphérique.

Seed et Idriss (1970) ont estimé le module $\left(\mathrm{K}_{2}\right)_{\max }$ tel que :

$$
\left(\mathrm{K}_{2}\right)_{\max }=3,5\left(\mathrm{I}_{\mathrm{D}}\right)^{\frac{2}{3}}
$$

\section{Equation du pieu et Ioi de réaction}

L'équation d'équilibre du tronçon de pieu soumis à un chargement réparti $p(z)$ (figure 2) s'écrit sous la forme :

$$
\begin{aligned}
& \frac{d T}{d z}=-p(z) \\
& T=\frac{d M}{d z} \\
& M=E I \frac{d^{2} y}{d z^{2}}
\end{aligned}
$$

Des relations (5), (6) et [7], on obtient l'équation d'équilibre du pieu :

$$
E I \frac{d^{4} y}{d z^{4}}+p(z)=0
$$




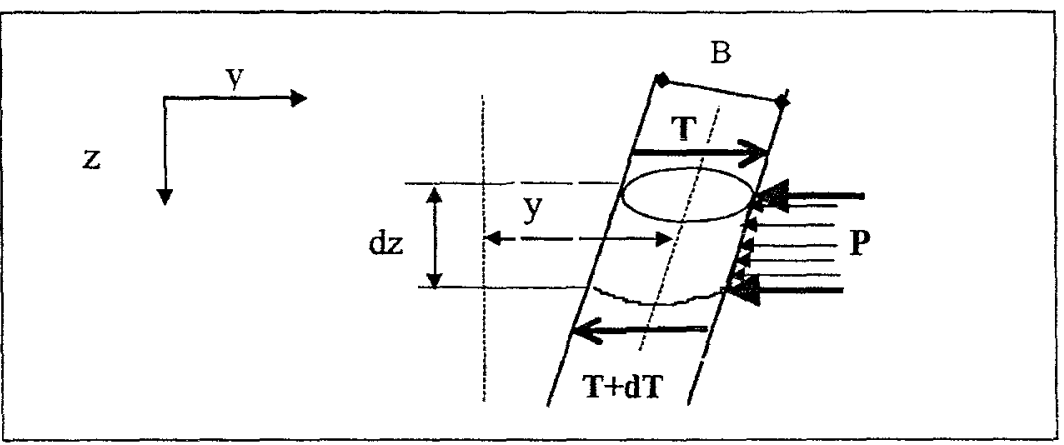

Figure 2: Schéma d'équilibre d'un tronçon du pieu

La loi de réaction đu sol avec prise en compte du cisaillement est :

$$
P=E_{s} y+P_{0}+G_{s} B \frac{d^{2} y}{d z^{2}}
$$

$\mathrm{P}_{0}$ : réaction initiale du sol.

L'équation différentielle du problème (8) devient alors :

$$
E I \frac{d^{4} y(z)}{d z^{4}}+G_{s} B \frac{d^{2} y(z)}{d z^{2}}+E_{s} y(z)=-P_{0}
$$

La solution de cette équation sans second membre, où $\mathbf{E}_{\mathbf{s}} \neq \mathbf{0}$, s'écrit :

$$
y=C_{1} e^{\frac{z}{z_{1}}} \cos \left(\frac{z}{z_{2}}\right)+C_{2} e^{\frac{z}{z_{1}}} \sin \left(\frac{z}{z_{2}}\right)+C_{3} e^{-\frac{z}{z_{1}}} \cos \left(\frac{z}{z_{2}}\right)+C_{4} e^{-\frac{z}{z_{1}}} \sin \left(\frac{z}{z_{2}}\right)
$$

avec

$$
z_{1}=\sqrt{\frac{4}{-\frac{G_{s} B}{E I}+\sqrt{\frac{4 E_{s}}{E I}}}} \quad \text { et } \quad z_{2}=\sqrt{\frac{4}{\frac{G_{s} B}{E I}+\sqrt{\frac{4 E}{E I}}}}
$$

La résolution numérique de l'équation différentielle, tenant compte du cisaillement et utilisant différentes lois de comportement du sol (efforts - déplacements) a été effectuée par la méthode matricielle dite « Matrice Transfert 》, appelée également la matrice de transmission.

\section{Etablissement de la matrice transfert de chaque tronçon}

En appelant $\left[\mathrm{K}_{\mathrm{n}}\right]$ la matrice transfert dont les termes sont notamment fonction de la longueur du tronçon $(\ell)$ et de sa rigidité $\mathrm{EI}$, on peut ainsi de manière générale, connaissant le vecteur $\left\{\mathrm{V}_{\mathrm{g}}\right\}$ à l'extrémité haute d'un tronçon, déterminer le vecteur $\left\{V_{d}\right\}$ de l'extrémité basse grâce à la relation suivante :

tel que

$$
\left\{\mathrm{V}_{\mathrm{d}}\right\}=\left[\mathrm{K}_{\mathrm{n}}\right] \times\left\{\mathrm{V}_{\mathrm{g}}\right\}
$$




$$
\left\{\mathrm{V}_{\mathrm{d}}\right\}=\left\{\begin{array}{l}
\mathrm{y}_{\mathrm{d}} \\
\mathrm{w}_{\mathrm{d}} \\
\mathrm{M}_{\mathrm{d}} \\
\mathrm{T}_{\mathrm{d}} \\
1
\end{array}\right\}\left[\mathrm{K}_{\mathrm{n}}\right]=\left[\begin{array}{lllll}
\mathrm{k}_{1} & \mathrm{k}_{2} & \mathrm{k}_{3} & \mathrm{k}_{4} & \mathrm{y}_{\mathrm{p}}(l) \\
\mathrm{k}_{5} & \mathrm{k}_{6} & \mathrm{k}_{7} & \mathrm{k}_{8} & \mathrm{w}_{\mathrm{p}}(l) \\
\mathrm{k}_{9} & \mathrm{k}_{10} & \mathrm{k}_{11} & \mathrm{k}_{12} & \mathrm{M}_{\mathrm{p}}(l) \\
\mathrm{k}_{13} & \mathrm{k}_{14} & \mathrm{k}_{15} & \mathrm{k}_{16} & \mathrm{~T}_{\mathrm{p}}(l) \\
0 & 0 & 0 & 0 & 1
\end{array}\right] \quad\left\{\mathrm{V}_{\mathrm{g}}\right\}=\left\{\begin{array}{l}
\mathrm{y}_{\mathrm{g}} \\
\mathrm{w}_{\mathrm{g}} \\
\mathrm{M}_{\mathrm{g}} \\
\mathrm{T}_{\mathrm{g}} \\
1
\end{array}\right\}
$$

avec

$k_{i / i=1 \ldots 16}$ sont fonction (EI, $\ell, E_{s}, G_{s}$ )

$y_{p}, w_{p}, M_{p}$ et $T_{p}$ sont fonction $\left(E I, \ell, E_{s}, G_{s}\right.$ et $P_{0}$ )

Un nouveau code de calcul appelé «CAPELA » a été développé. Ce code, réalisé en Visual Basic, a pour objet le calcul des déplacements et des efforts d'un pieu isolé soumis à des efforts latéraux en tout point le long de celui-ci, avec prise en compte du cisaillement à l'interface des couches du sol.

La validation de ce modèle est faite d'abord en comparant les résultats obtenus (sans prise en compte du cisaillement) avec ceux issus du programme «PILATE » (Frank et al. 1990) et d'autre part, en confrontant les résultats de calcul (avec et sans prise en compte du cisaillement) aux résultats expérimentaux des essais sur des modèles de pieux réduits centrifugés.

\section{Essai sur des modèles réduits centrifugés}

Une campagne d'essais sur des modèles réduits de pieux chargés latéralement en centrifugeuse a été réalisée au L.C.P.C de Nantes par Mezazigh (1995). L'objectif était la détermination de l'effet de présence d'un taius sur les courbes de réaction p-y. Au total 59 essais de pieux chargés latéralement dans un massif sableux ont été effectués. Les pieux utilisés sont considérés comme longs et flexibles. Les paramètres pris en compte dans ces essais sont : la pente du talus, la position du pieu par rapport à la crête du talus et la densité du sol. Dans cette présentation seuls les pieux isolés implantés dans un sol horizontal (sans talus), soumis à la charge latérale sont considérés. Dans la présente étude, nous prenoñs les caractéristiques des pieux et les courbes de réaction $\mathrm{p}-\mathrm{y}$ du sol déterminées expérimentalement (Mezazigh, 1995) comme données de calcul pour les logiciels CAPELA et PILATE.

\section{Le massif du sol}

Le sol utilisé est du sable, un matériau siliceux propre (sable de Fontainebleau), couramment utilisé lors d'essais en vraie grandeur ou à échelle réduite. C'est un sable dont environ $70 \%$ des éléments ont un diamètre inférieur à $200 \mu \mathrm{m}$. Dans les quatre exemples de calculs réalisés avec PILATE et CAPELA, le sol a un poids volumique d'environ $16.19 \mathrm{kN} / \mathrm{m}^{3}$ pour un sable dense et environ $15 \mathrm{kN} / \mathrm{m}^{3}$ pour un sable moyennement dense ce qui correspond respectivement à des indices de densité de 0,81 et 0,63 . 


\section{Le pieu modèle}

Le pieu modèle est un tube en aluminium $\mathrm{AU} 4 \mathrm{G}$, de diamètre extérieur de $18 \mathrm{~mm}$, d'épaisseur de $1,5 \mathrm{~mm}$ et de longueur de $380 \mathrm{~mm}$. La fiche est de $300 \mathrm{~mm}$. L'accélération centrifuge de $40 \mathrm{~g}$ permet de simuler un pieu prototype de $720 \mathrm{~mm}$ de diamètre et fiché de $12 \mathrm{~m}$. Le module de rigidité est de $51,4 \mathrm{MN} . \mathrm{m}^{2}$.

L'instrumentation des pieux permet d'effectuer des mesures directes des déplacements en tête et des moments fléchissants le long du fût du pieu pour chaque niveau de chargement. Par contre, les déformées et les pressions le long du pieu sont obtenues par une double intégration et une double dérivation des courbes des moments fléchissants mesurés.

\section{Validation et analyse des résultats}

Dans le but de valider le programme CAPELA, une étude comparative a été menée sur les résultats obtenus par PILATE en utilisant les exemples tirés des essais effectués sur les modèles réduits centrifugés. Les données utilisées dans les différents exemples traités se basent sur les caractéristiqués mécaniques et géométriques du pieu et du sol des essais centrifugés. Les résultats obtenus par les deux logiciels ont montré une bonne concordance (figure 3). L'écart entre les déplacements en tête et les moments maximaux obtenus par les deux logiciels ne dépasse pas $6 \%$. On a procédé ensuite à une comparaison des courbes expérimentales des moments et des déplacements, obtenues par les essais sur les modèles réduits centrifugés (Mezazigh, 1995) avec celles obtenues par le calcul avec et sans prise en compte du cisaillement. L'écart entre les déplacements en tête et les moments maximaux obtenus a dépassé respectivement le $27 \%$ et $25 \%$ (Figure 4). La prise en compte du cisaillement dans le logiciel a diminué cet écart (le nouveau écart obtenu est de $10 \%$ et de 6\%) (Figure 5). Les courbes de déplacements et de moments obtenues dans ce cas par CAPELA s'accordent mieux avec celles des essais. Ainsi, l'influence du cisaillement à l'interface des couches du sol sur le comportement du pieu chargé latéralement est mise en évidence.

\section{Conclusion}

Le programme CAPELA est un code de calcul de pieu chargé latéralement basé sur la méthode au module de réaction et s'appuyant sur une méthode de calcul souple qui est la méthode de matrice transfert. Par ailleurs, ce programme a été doté d'un menu d'introduction de données élégant et facile d'utilisation.

La méthode simple et rapide de calcul appelée «matrice transfert» permet de donner la distribution des moments et des déplacements le long d'un pieu chargé latéralement. Cette méthode présente un intérêt pour le dimensionnement du pieu lui même.

Une étude comparative des résultats de calcul de pieux chargés latéralement obtenus par PLATE, CAPELA et des essais sur des modèles réduits centrifugés a été effectuée. Cette étude a un double objectif, d'abord de montrer l'utilité de la prise en compte du cisaillement à l'interface des couches du sol, d'autre part la validation du programme CAPELA. Cette simple comparaison des résultats permet 
donc d'avoir une estimation concrète et globale de l'effet du cisaillement à l'interface des couches de sol et mettre en évidence l'influence de cisaillement sur le comportement du pieu chargé latéralement.

Un autre avantage de ce code est qu'il est adapté à l'étude des pieux chargés latéralement en $n^{\prime}$ importe quel point le long du pieu et n'importe quelles conditions environnementales (présence de talus par exemple).

\section{Références}

[1] R.Frank, J.L. Bangratz ef M. Kutniak (1990), PILATE-LCPC : Programme de calcul d'un pieu isolé soumis à des efforts de flexion en tête et à des poussées latérales de sol. Laboratoires des Ponts et Chaussés, Paris, 69p.

[2] S. Mezazigh (1995). Etude expérimentale des pieux chargés latéralement: proximité d'un talus et effet de groupe. Thèse de doctorat de l'Université et de l'Ecole Centrale de Nantes, $217 \mathrm{p}$.

[3] P.L. Pasternak (1954). On a new method of analysis of an elastic foundation by means of two foundation constants. Gosudarstvennoe Izdatelstro Liberatuni po Stroitelsturi Arkhitekture, Moscow.

[4] Seed H.B. et Idriss I.M. (1970). Soil moduli and damping factors for dynamic rsponse analysis. Report UCB/EERC - 70/10, University of California, Berkeley.

[5] Seed H.B., Wong R.T.,. Idriss I.M, et Tokimatsu K.(1986). Moduli and damping factors for dynamic analyses of cohesionless soils. ASCE Journal of Geotechnical Engineering, 112, p. 1016-1032.

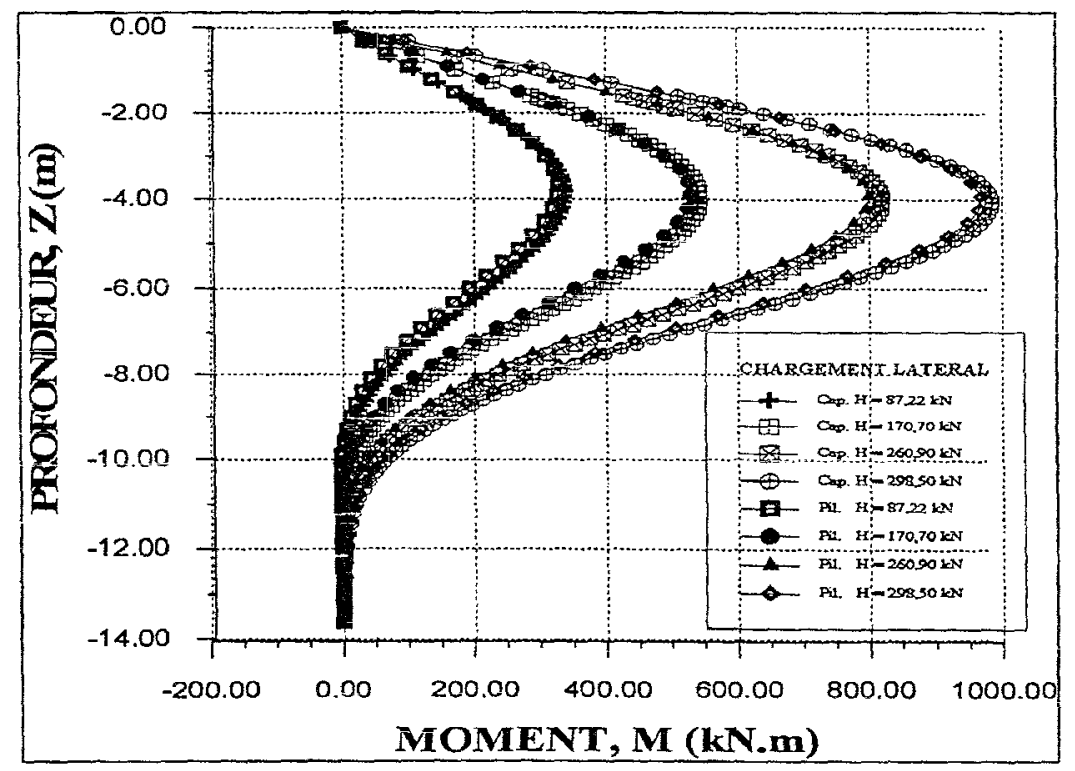

Figure 3 : Comparaison entre les résultats obtenus à l'aide de CAPELA et ceux obtenus par PILATE 


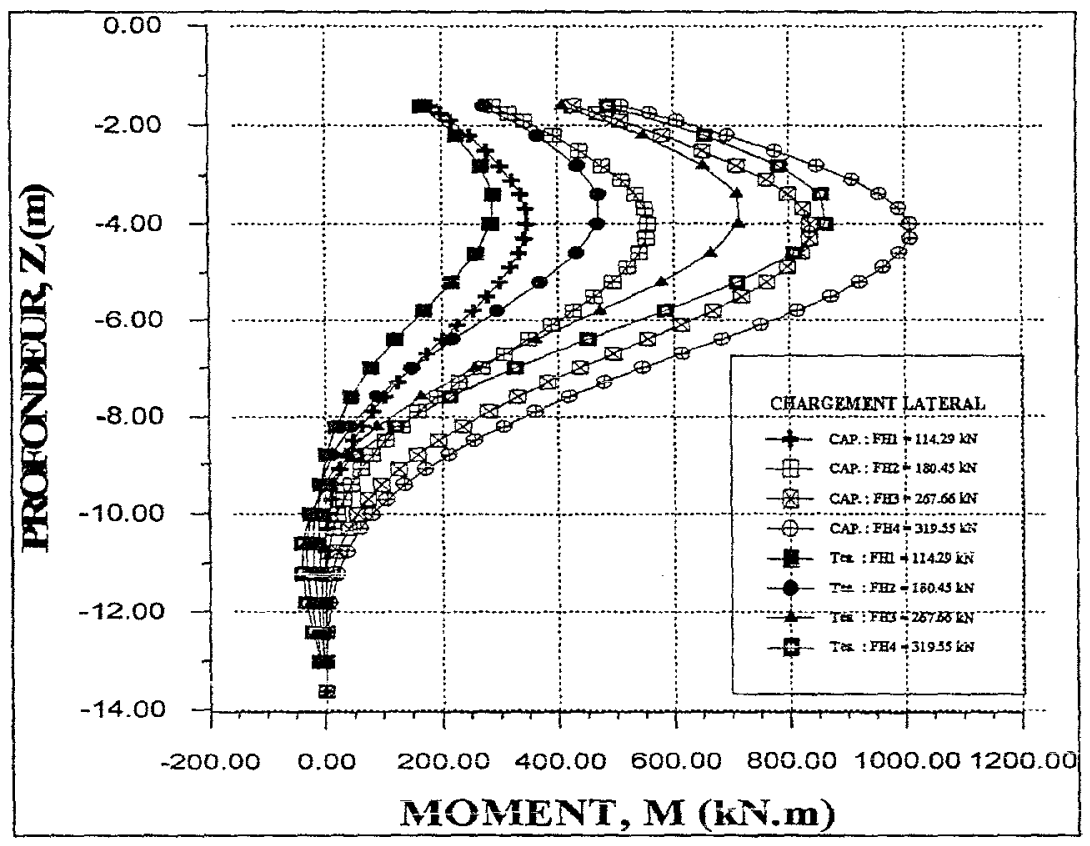

Figure 4 : Comparaison des courbes expérimentales à celles obtenues par CAPELA (sans prise en compte du cisaillement).

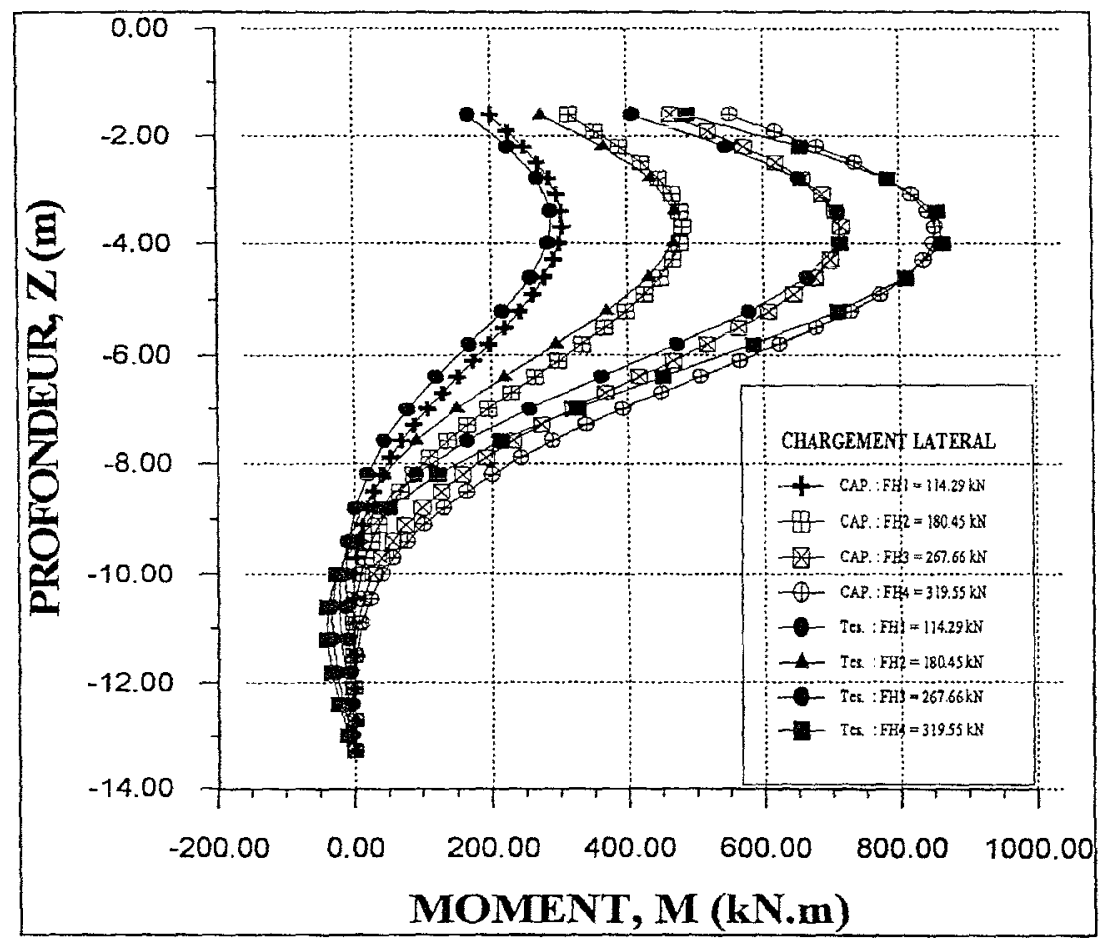

Figure 5 : Comparaison des courbes expérimentales à celles obtenues par CAPELA (ovec prise en compte du cisaillement) 